\title{
Meeting abstracts from the 11th European Cytogenetics Conference
}

\author{
Florence, Italy. 01-04 July 2017 \\ Published: 29 June 2017

\section{Invited Lecture Abstracts} \\ L1 \\ Chromosomes to Circulating DNA \\ Yuk Ming Dennis Lo \\ The Chinese University of Hong Kong Li Ka Shing Institute of Health \\ Sciences, Hong Kong, China \\ Molecular Cytogenetics 2017, 10(Suppl 1):L1
}

In recent years, there have been an intense interest in the diagnostic applications of circulating DNA. One source of such interest is in the rapid adoption of noninvasive prenatal testing (NIPT) using cell-free fetal DNA in maternal plasma. One area of focus of my laboratory is to explore the limit of NIPT. In this regard, we have recently completed a 'second generation' noninvasive fetal genome from maternal plasma. For this work, we have sequenced the plasma DNA of a pregnant woman to a depth of 270X haploid genome coverage. This represents the deepest that a single plasma DNA sample has been sequenced to date. Using this approach, together with a novel bioinformatics pipeline, we are able to deduce, for the first time, fetal de novo mutations on a genomewide level with a sensitivity of $85 \%$ and a positive predictive value of $74 \%$. We are also able to determine the maternal inheritance of the fetus with a 90 -fold increase in resolution when compared with previous attempts. Finally, we have shown that plasma DNA molecules have preferred ending sites. Interestingly, fetal-derived and maternal-derived plasma DNA molecules have different sets of such preferred ending sites. This latter discovery has opened up many new avenues of investigation and has created new applications, e.g. for determining the fraction of fetal DNA without using genetic polymorphisms or DNA methylation markers. NIPT also serves as a model for developing noninvasive diagnostics in many other fields, e.g. oncology and transplantation.

\section{L2}

Genomic and functional overlap between somatic and germline chromosomal rearrangements

Wigard Kloosterman

University Medical Center Utrecht, Utrecht, The Netherlands

Molecular Cytogenetics 2017, 10(Suppl 1):L2

Structural genomic variants form a common type of genetic alteration underlying human genetic disease and phenotypic variation. Despite major improvements in genome sequencing technology and data analysis, the detection of structural variants still poses challenges, particularly when variants are of high complexity. Emerging long-read single- molecule sequencing technologies provide new opportunities for detection of structural variants. We demonstrate sequencing of the genomes of two patients with congenital abnormalities using the ONT
MinION at $11 x$ and $16 x$ mean coverage, respectively. We developed a bioinformatic pipeline - NanoSV - to efficiently map genomic structural variants (SVs) from the long-read data. Using NanoSV, we readily detected all de novo rearrangements involving multiple chromosomes originating from complex chromothripsis events. Genome-wide surveillance of SVs, revealed 3,253 (33\%) novel variants that were missed in short-read data of the same sample, the majority of which are duplications $<200 \mathrm{bp}$ in size. Long sequencing reads enabled efficient phasing of genetic variations, allowing the construction of genomewide maps of phased SVs. We employed read-based phasing to show that all de novo chromothripsis breakpoints occurred on paternal chromosomes and we resolved the long-range structure of the chromothripsis. Our work demonstrates the value of long-read sequencing for genetic analyses in life sciences research and clinical diagnostics.

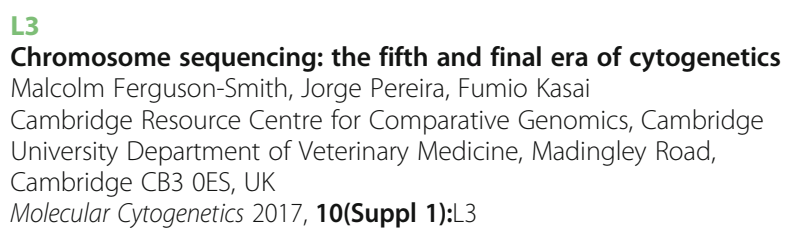

The modern history of cytogenetics fits into five eras; chromosome spreading, chromosome banding, chromosome painting, chromosome arraying and now chromosome sequencing. T.C. Hsu, the centenary of whose birth we celebrate, was a pioneer of the first. His use of hypotonic fluid to spread chromosomes was key to the emergence of human cytogenetics and to the collection of animal karyotypes published in his Atlas of Mammalian Chromosomes edited with Kurt Benirschke. Forty-five years later, chromosome sequencing achieved the ultimate resolution by defining chromosome disease in terms of base pairs. It relies on sorting and collecting chromosomes in fluid suspension by flow cytometry and, like chromosome painting, on DNA amplification of the sorted samples. Chromosome sorting is currently the most precise method used for measuring chromosome and genome size. Ten thousand cleanly-sorted chromosomes provide sufficient DNA for Next Generation sequencing in both plants and animals. Our collaborations demonstrate the potential of this approach. For example, sequence from sorted gorilla $Y$ chromosomes reveal, remarkably, that human and gorilla Ys are similar and that chimpanzee has lost half the $Y$ genes present in both the other species. In two species of anole lizards, one with large heteromorphic sex chromosomes and the other with small homomorphic sex chromosomes, sequence from the ancestral $Y$ chromosome has been fused with sequence from three microchromosomes to form a large $\mathrm{Y}$ chromosome. Sequencing B chromosomes in two deer species demonstrate the inclusion of different 
biomarkers. There were also features of convergent evolution in branches of evolutionary trees, including trisomies and monosomies, as well as TP53 mutations in Wilms tumours, CDKN2A deletions in neuroblastoma, and CDK4 amplifications in rhabdomyosarcoma. Subclones were the major arena of genome evolution. We observed four major trajectories of cancer cell evolution including (1) subclonal variation, (2) subclone coexistence, (3) subclonal explosions, and (4) clonal sweeps. These trajectories exhibited a striking correlation to clinical risk groups. Conclusion

Even in very young patients, cancer is a genetically dynamic disease over space and time. On the one hand, intratumour diversity is a significant source of error in genetic biomarker assessment. On the other hand, specific evolutionary patterns may be useful as future clinical predictors.

\section{9}

Novel recurrent chromosome anomalies in Shwachman Diamond syndrome

Emanuela Maserati ${ }^{1}$, Roberto Valli ${ }^{1}$, Lucia Nacci ${ }^{2}$, Annalisa Frattini ${ }^{3}$, Federico Grilli ${ }^{1}$, Francesco Pasquali

${ }^{1}$ University Of Insubria, Department of Medicine And Surgery, Varese, Italy; ${ }^{2}$ University of Pavia, Department of Molecular Medicine, Pavia, Italy; ${ }^{3}$ National Council of Research, Irgb, Milano, Italy

Correspondence: Emanuela Maserati

Molecular Cytogenetics 2017, 10(Suppl 1):09

Clonal chromosome anomalies are frequently acquired in the bone marrow (BM) of patients with Shwachman-Diamond syndrome (SDS), and two are the most frequent: an isochromosome of the long arm of chromosome $7, \mathrm{i}(7)(\mathrm{q} 10)$, and an interstitial deletion of the long arm of chromosome 20, del(20)(q). Patients with SDS have a risk of developing myelodysplasia (MDS) and/or acute myeloid leukaemia (AML), and the presence of chromosome changes was studied in relation with this risk. Starting in 1999 we have monitored the cytogenetic picture of a cohort of 92 Italian patients with SDS by all suitable cytogenetic and molecular methods. Clonal anomalies in BM were present in 41/92 patients. The $\mathrm{i}(7)(\mathrm{q} 10)$ was observed in 16 patients, and the del(20)(q) in 15 , both these changes in four, but in independent clones. So, the most frequent clonal anomalies were found in 35 patients. Other, different, clonal anomalies were found in the BM of 13 patients, in eight cases in the absence of $\mathrm{i}(7)(\mathrm{q} 10)$ or del(20)(q), in five cases in association with one of these changes. In these less common clonal anomalies, the distribution of the chromosomes involved was markedly disparate, and some of them were novel and recurrent:

- structural rearrangements of chromosome 7, mainly unbalanced (deletions, inversions or translocations), were present in five of our 13 patients, three of whom developed MDS/AML.

- a further complex rearrangement of the more common del(20)(q), leading to duplicated and deleted portions, was identical in two patients, with almost identical a-CGH profiles, neither developed MDS/AML.

- an unbalanced translocation $\mathrm{t}(3 ; 6)$, with partial trisomy of the long arm of chromosome 3 and partial monosomy of the long arm of chromosome 6 , was not identical but very similar in two patients, one of whom developed MDS/AML.

\section{0}

Deletion $13 q$ characterised by SNP microarray profiling of a large cohort of CLL patients

Nicole Chia ${ }^{1}$, Dr Melody Caramins ${ }^{2}$

${ }^{1}$ Qml Pathology, Genetics, Murrarrie, Australia; ${ }^{2}$ Sds Pathology, Genomic Diagnostics, Sydney, Australia

Correspondence: Nicole Chia

Molecular Cytogenetics 2017, 10(Suppl 1):010

The investigation of Chronic Lymphocytic Leukemia (CLL) by conventional cytogenetic analysis combined with fluorescence in-situ hybridisation (FISH) has been regarded as the gold standard. Detection and characterisation of non-random rearrangements is hindered in part by the limitation of cell culturing and the targeted approach of FISH testing. More recently the implementation of molecular karyotyping by high resolution Single Nucleotide Polymorphism (SNP) microarray platforms has augmented the investigation protocol of haematological malignancies in diagnostic pathology.

Deletion $13 q$ is reported in up to $50 \%$ of patients with CLL and has historically been reported in association with low risk disease. Molecular karyotyping has revealed the diversity and complexity of rearrangements within the del13q region. This has culminated in the classification of the 13q deletion into type I (exclusive of Rb1) and type II (inclusive of Rb1). However uncertainty exists with regard to the prognostic significance, particularly with respect to the large, type II deletion.

To evaluate this we investigated the molecular karyotype of $500 \mathrm{CLL}$ patients. We ascertained the incidence of del13q type I and type II, complexity of deletions, association with additional karyotypic changes and $\mathrm{cnLOH}$ chromosome 13 . Our results show that there is no significant difference in the incidence or association with additional karyotypic changes, but type II deletion is associated with complex 13q deletions $(p=0.0001)$. Furthermore there is no significant difference with respect to cytogenetic progression in follow-up testing of 43 patients. This study highlights the benefit of SNP microarray analysis in characterising deletion anatomy and the need for re-evaluation of risk stratification of non-random rearrangements.

\section{1}

The transcriptome plasticity of genome amplification in cancer Gemma Macchia ', Alberto L'abbate ', Doron Tolomeo ', Grazia Visci 1 Giulia Daniele 1, Angelo Lonoce ', Lucia Anna Muscarella 2,

Clelia Tiziana Storlazzi

'University of Bari "aldo Moro", Biology, Bari, Italy; ${ }^{2}$ Irccs Casa Sollievo Della Sofferenza, Laboratorio Di Genetica Medica, San Giovanni Rotondo, Italy

Correspondence: Gemma Macchia

Molecular Cytogenetics 2017, 10(Suppl 1):011

Genome amplification, in the form of homogeneously staining regions, double minutes, and ring/giant rod-shaped markers, is a pivotal event in many tumors. It was recently shown that amplifications as extrachromosomal DNA are present in nearly half of all tumors, representing a driving force towards their accelerated evolution. To achieve a better understanding of the implications of genomic amplifications we focused on their structure and impact upon transcription. Amplified cancer-associated genes are often overexpressed as a direct consequence of the copy number gain. We analyzed the whole genome (WGS) and transcriptome (RNA-seq) sequencing data of nine small cell lung carcinoma (SCLC), seven neuroblastoma (NB) and three welldifferentiated liposarcoma (WDLPS) cell lines, all carrying genomic amplifications. A widespread heterogeneity of the amplicon arrangement was detected in most of the samples, disclosing the progressive evolution of their structure through cell divisions. By integrating the WGS (structural variation calling) and RNA-seq (chimeric transcripts detection) data, we detected a burst of chimeras derived from posttranscriptional events (i.e. cis- or trans-splicing) involving amplified genes. Notably, we found PVT1 (8q24) and RLF (1p34.2) as hotspots for cis- or trans-splicing events in SCLC and NB samples with MYC and MYCL1 amplifications, respectively. In WDLPS cell lines, we found extremely complex fusion genes, such as those involving three partners or assembled by multiple interposed non-contiguous, non-collinear genomic fragments (spliced out in the mature transcript). Our results strongly indicate that the "amplification/overexpression" paradigm does not cover all aspects of the genomic amplification impact upon transcription. The extraordinary transcriptome plasticity herein described, enriching the genetic repertoire of cancer cells with genomic amplifications, likely provides a selective advantage and might have a crucial role in cancer establishment and progression. 